\title{
Gross hematuria in a boy — "spot" the diagnosis:Questions
}

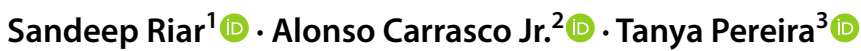 \\ Received: 16 December 2021 / Revised: 20 December 2021 / Accepted: 21 December 2021 / Published online: 3 February 2022 \\ (c) The Author(s), under exclusive licence to International Pediatric Nephrology Association 2022
}

Keywords Child · Gross hematuria $\cdot$ Recurrent

\section{Case}

A 9-year-old male presented to Nephrology clinic with intermittent painless gross hematuria for 2 months. Urine color was reported as light pink to dark red without clots. He had no other urinary symptoms except for frequency a month prior to the onset of hematuria that resolved spontaneously after several days. There was no fever or acute illness with the hematuria or in the 2 months preceding it. Two days prior to onset of hematuria, he was noted to have a bruise over mid back attributed to a playground injury. He had chronic constipation which was well controlled with daily polyethylene glycol. His past medical history was significant for hypospadias surgery during infancy, attention deficit hyperactivity disorder, generalized anxiety disorder, and oppositional defiance disorder. $\mathrm{He}$ had no history of urinary tract infections. Home medications included cetirizine, methylphenidate, vitamin D, fluoxetine, multivitamins, and polyethylene glycol. There was no known family history of kidney disease, nephrolithiasis, bleeding, or clotting disorder. Family history for biological father was not available and limited for biological mother who was adopted.

He was evaluated by his pediatrician 2 days after onset of hematuria and noted to have normal blood pressure. His urinalysis was unremarkable with $\mathrm{pH} 7$, specific gravity 1.024 , amorphous

The answers to these questions can be found at https://doi.org/10. 1007/s00467-022-05461-0.

Sandeep Riar

sandeepriar.md@gmail.com

1 Department of Pediatrics, Nephrology Division, Children's Mercy Hospital, 2401 Gillham Road, Kansas City, MO 64108, USA

2 Department of Surgery, Urology Division, Children's Mercy Hospital, 2401 Gillham Road, Kansas City, MO 64108, USA

3 Department of Pediatrics, Nephrology Division, Maria Fareri Children's Hospital, New York Medical College, Boston Children's Health Physicians, Valhalla, NY 10595, USA sediment with negative heme/protein/leukocyte esterase/nitrite, and $0-2 \mathrm{RBCs} / \mathrm{hpf}$. Urine culture and hypercalciuria screen were negative. A blood test showed normal kidney function, bicarbonate, calcium, and $\mathrm{C} 3$ complement. A kidney ultrasound obtained approximately 6 weeks after the onset of hematuria showed mild circumferential bladder wall thickening, attributed to incomplete distention, cystitis, or neurogenic bladder.

Evaluation in Nephrology clinic showed normal growth parameters and normal blood pressure. He had no abdominal or costovertebral tenderness, and genital examination was unremarkable. Urinary dipstick was negative with $\mathrm{pH} 8$ and specific gravity 1.008 . There was no hypercalciuria or hyperuricosuria. Abdominal X-ray showed mild stool retention with no changes to suggest stool impaction.

\section{Questions:}

1. What additional question and complementary finding on physical exam might indicate non-glomerular rather than glomerular cause of gross hematuria?

2. What is the differential diagnosis of gross hematuria with this clinical feature?

3. What is the most likely cause for this patient's presentation?

4. What is the significance of absence of hematuria on urine studies?

\section{Declarations}

Competing interests We confirm that none of us have any competing interests to declare that are relevant to the content of this article.

Publisher's note Springer Nature remains neutral with regard to jurisdictional claims in published maps and institutional affiliations. 